\title{
ON THE DEFINITION OF THE DUAL LIE COALGEBRA OF A LIE ALGEBRA
}

\author{
BERTIN DiARRA
}

\begin{abstract}
Let $L$ be a Lie algebra over a field $K$. The dual Lie coalgebra $L^{\circ}$ of $L$ has been defined by W. Michaelis to be the sum of all good subspaces $V$ of the dual space $L^{*}$ of $L: V$ is good if ${ }^{t} m(V) \subset$ $V \otimes V$, where $m$ is the multiplication of $L$. We show that $L^{\circ}=$ ${ }^{t} m^{-1}\left(L^{*} \otimes L^{*}\right)$ as in the associative case.
\end{abstract}

Let $L$ be a Lie algebra over the field $K$ with multiplication $m: L \otimes L \rightarrow$ $L$ : i.e. $m$ is a linear map and setting $m(x \otimes y)=[x, y]$, one has

$$
[x, x]=0
$$

$$
[x,[y, z]]+[z,[x, y]]+[y,[z, x]]=0
$$

Let $L^{*}$ be the dual vector space of $L$ and ${ }^{t} m: L^{*} \rightarrow(L \otimes L)^{*}$ be the transpose of $m$. We identify $L^{*} \otimes L^{*}$ with a subspace of $(L \otimes L)^{*}$ and we set $L^{\odot}={ }^{t} m^{-1}\left(L^{*} \otimes L^{*}\right)$.

Fix $f \in L^{*}$ and consider the linear map $\gamma_{f}: L \rightarrow L^{*}$ defined by

$$
\left\langle\gamma_{f}(x), y\right\rangle=\langle f,[x, y]\rangle=\left\langle{ }^{t} m(f), x \otimes y\right\rangle, \quad x, y \in L
$$

Setting, as usual, $a d x(y)=[x, y]$, one has $\gamma_{f}(x)={ }^{t}(a d x)(f)$. Sometimes, we shall write $\gamma_{f}(x)=x \cdot f$. 
Lemma 1. For $f \in L^{*}$, the following statements are equivalent

(i) $f \in L^{\odot}$

(ii) The linear map $\gamma_{f}: L \rightarrow L^{*}$ is of finite rank.

Proof: The equivalence follows readily from (3).

Moreover, since $L^{*} \otimes L^{*}$ can be identified with the space of the linear maps of $L$ into $L^{*}$ of finite rank, via $\iota: L^{*} \otimes L^{*} \hookrightarrow \operatorname{Hom}\left(L, L^{*}\right)$ by setting $\iota(f \otimes g)(x)=f(x) g$, one has $f \in L^{\odot}$ iff $\gamma_{f}=\sum_{j=1}^{n} f_{j} \otimes g_{j}$ iff ${ }^{t} m(f)=\sum_{j=1}^{n} f_{j} \otimes g_{j}$

Lemma 2. For $f \in L^{\odot}$ and $x \in L$, one has, $\gamma_{f}(x)=x \cdot f \in L^{\odot}$. Moreover $\gamma_{f}(L)$ is a vector subspace of $L^{\odot}$ of finite dimension.

Proof: If $f \in L^{\odot}$, then for $x, y \in L$, one has $\langle f,[x, y]\rangle=\left\langle\gamma_{f}(x), y\right\rangle=$ $\sum_{j=1}^{n}\left\langle f_{j}, x\right\rangle\left\langle g_{j}, y\right\rangle$. However, (2) can be written $[x,[y, z]]=-[y,[z, x]]-$ $[z,[x, y]]$. Therefore, one has

$$
\begin{aligned}
\left\langle\gamma_{x} \cdot f(y), z\right\rangle & =\langle x \cdot f,[y, z]\rangle \\
& =\langle f,[x,[y, z]]\rangle \\
& =-\langle f,[y,[z, x]]\rangle-\langle f,[z,[x, y]]\rangle \\
& =-\sum_{j=1}^{n}\left\langle f_{j}, y\right\rangle\left\langle g_{j},[z, x]\right\rangle-\sum_{j=1}^{n}\left\langle f_{j}, z\right\rangle\left\langle g_{j},[x, y]\right\rangle \\
& =\sum_{j=1}^{n}\left\langle f_{j}, y\right\rangle\left\langle x \cdot g_{j}, z\right\rangle-\sum_{j=1}^{n}\left\langle x \cdot g_{j}, y\right\rangle\left\langle f_{j}, z\right\rangle .
\end{aligned}
$$

Hence $\gamma_{x \cdot f}(y)=\sum_{j=1}^{n}\left\langle f_{j}, y\right\rangle x \cdot g_{j}-\sum_{j=1}^{n}\left\langle x \cdot g_{j}, y\right\rangle f_{j}$. It follows that $\gamma_{x \cdot f}$ is of finite rank, that is $x \cdot f=\gamma_{f}(x) \in L^{\odot}$. Then, it is clear that $\gamma_{f}(L)$ is a vector subspace of $L^{\odot}$ and finite dimensional. 
Note. One deduces from the above proof that if $f \in L^{\odot}$ and ${ }^{t} m(f)=$ $\sum_{j=1}^{n} f_{j} \otimes g_{j}$ then for $x \in L$, one has

$$
{ }^{t} m(x \cdot f)=\sum_{j=1}^{n} f_{j} \otimes\left(x \cdot g_{j}\right)-\sum_{j=1}^{n}\left(x \cdot g_{j}\right) \otimes f_{j}
$$

Theorem 1. $L^{\odot}$ is a good subspace of $L^{*}$ i.e. ${ }^{t} m\left(L^{\odot}\right) \subset L^{\odot} \otimes L^{\odot}$. Moreover, one has $L^{\odot}=L^{\circ}$.

Proof: Let $f \in L^{\odot}$ and let $\left(g_{j}\right)_{1 \leq j \leq n}$ be a base of $\gamma_{f}(L) \subset L^{\odot}$. One has $g_{j}=x_{j} \cdot f$ and for any $x \in L, \gamma_{f}(x)=\sum_{j=1}^{n} f_{j}(x) g_{j}$; hence ${ }^{t} m(f)=$ $\sum_{j=1}^{n} f_{j} \otimes g_{j}, f_{j} \in L^{*}$. However $m=-m \circ \tau$ (skew-symmetry), therefore, one has ${ }^{t} m=-{ }^{t} \tau \circ{ }^{t} m$ and ${ }^{t} m(f)=\sum_{j=1}^{n} f_{j} \otimes g_{j}=-{ }^{t} \tau\left(\sum_{j=1}^{n} f_{j} \otimes g_{j}\right)=$ $-\sum_{j=1}^{n} g_{j} \otimes f_{j}$. Since $\left(g_{j}\right)_{1 \leq j \leq n}$ is free in $L^{*}$, there exists for $1 \leq \ell \leq n$ $y_{\ell} \in L$ such that $\left\langle g_{j}, y_{\ell}\right\rangle=\delta_{j \ell}$. Hence $\left(y_{\ell} \otimes 1_{L^{*}}\right)\left({ }^{t} m(f)\right)=\sum_{j=1}^{n}\left\langle f_{j}, y_{\ell}\right\rangle g_{j}=$ $-\sum_{j=1}^{n}\left\langle g_{j}, y_{\ell}\right\rangle f_{j}=-f_{\ell}$, that is $f_{\ell}=-\sum_{j=1}^{n}\left\langle f_{j}, y_{\ell}\right\rangle g_{j} \in \gamma_{f}(L)$. It follows that ${ }^{t} m(f)=\sum_{j=1}^{n} f_{j} \otimes g_{j} \in \gamma_{f}(L) \otimes \gamma_{f}(L) \subset L^{\odot} \otimes L^{\odot}$ and $L^{\odot} \subset L^{\circ}$.

On the other hand, it is clear that any good subspace $V$ of $L^{*}$ is contained in $L^{\odot}$, therefore $L^{\circ} \subset L^{\odot}$. We have proved that $L^{\odot}=L^{\circ}$.

Note. If $f \in L^{\odot}$ and if $\left(x_{j} \cdot f\right)_{1 \leq j \leq n}$ is a base of $\gamma_{f}(L)$, one has ${ }^{t} m(f)=\sum_{j=1}^{n}\left(y_{j} \cdot f\right) \otimes\left(x_{j} \cdot f\right)$ where, for $1 \leq j \leq n, y_{j}$ is such that $\left\langle f,\left[x_{\ell}, y_{j}\right]\right\rangle=\delta_{\ell j}$.

Furthermore, if $x \in L$, one has

(4) ${ }^{t} m(x \cdot f)=\sum_{j=1}^{n}\left(y_{j} \cdot f\right) \otimes\left[x \cdot\left(x_{j} \cdot f\right)\right]-\sum_{j=1}^{n}\left[x \cdot\left(x_{j} \cdot f\right)\right] \otimes\left(y_{j} \cdot f\right)$. 
Remark. Put $\Delta=\left.{ }^{t} m\right|_{L \odot}: L^{\odot} \rightarrow L^{\odot} \otimes L^{\odot}$. Following W. Michaelis $[\mathbf{1}]$, (see also $[\mathbf{2}],[\mathbf{3}],[\mathbf{4}],[\mathbf{5}]$ and $[\mathbf{6}]$ ) one obtains a Lie coalgebra $\left(L^{\odot}, \Delta\right)$, that is :

$$
\Delta=-\tau \circ \Delta
$$

if the characteristic of $K$ is different from 2 and $\operatorname{Im} \Delta \subset \operatorname{Im}\left(1_{L} \odot-\tau\right)$ otherwise $[\tau(f \otimes g)=g \otimes f]$.

$$
\left(i d_{3}+\sigma+\sigma^{2}\right) \circ\left(1_{L \odot} \otimes \Delta\right) \circ \Delta=0
$$

where $\sigma(f \otimes g \otimes h)=h \otimes f \otimes g$.

This follows from (1) and (2). Notice that (2) is equivalent to $m \circ$ $\left(1_{L} \otimes m\right) \circ\left(i d_{3}+\rho+\rho^{2}\right)=0$ where $\rho(x \otimes y \otimes z)=z \otimes x \otimes y$ and one has $\left.{ }^{t} \rho^{2}\right|_{L \odot}=\sigma$.

For $A \subset L^{\odot}$, let $\operatorname{span}(A)$ be the vector subspace of $L^{\odot}$ spaned by $A$.

Theorem 2. Let $f \in L^{\odot}$. Put

$$
\begin{aligned}
& V_{0}=K \cdot f \\
& V_{1}=\gamma_{f}(L)=\left\{x_{1} \cdot f, x_{1} \in L\right\} \\
& V_{2}=\operatorname{span}\left\{x_{2} \cdot f_{1}, x_{2} \in L, f_{1} \in V_{1}\right\} \\
& \ldots \ldots \ldots \ldots \ldots \ldots \ldots \ldots \ldots \ldots \ldots \ldots \ldots \ldots \ldots \ldots \ldots \ldots \ldots \ldots \ldots \ldots \ldots \ldots \ldots \ldots \ldots \ldots \ldots \ldots \ldots \ldots \ldots \ldots \\
& V_{n}=\operatorname{span}\left\{x_{n} \cdot f_{n-1}, x_{n} \in L, f_{n-1} \in V_{n-1}\right\}
\end{aligned}
$$

Then $W=\sum_{n \geq 0} V_{n}$ is a Lie subcoalgebra of $L^{\odot}$ and is the smallest Lie subcoalgebra of $L^{\odot}$ that contains $f$.

Proof: We have seen that if $f \in L^{\odot}$, then $\Delta(f)=\sum_{j=1}^{n}\left(y_{j} \cdot f\right) \otimes\left(x_{j} \cdot f\right)$. It follows that $\Delta\left(V_{0}\right) \subset V_{1} \otimes V_{1}$. Furthermore $V_{1} \subset L^{\odot}$, and by induction one has $V_{n} \subset L^{\odot}$. On the other hand, if $x_{n} \in L, f_{n-1} \in V_{n-1}, n \geq 1$, one has by (4)

$$
\begin{aligned}
& \Delta\left(x_{n} \cdot f_{n-1}\right)=\sum_{j=1}^{m}\left(y_{n j} \cdot f_{n-1}\right) \otimes\left[x_{n} \cdot\left(x_{n j} \cdot f_{n-1}\right)\right] \\
& \quad-\sum_{j=1}^{m}\left[x_{n} \cdot\left(x_{n j} \cdot f_{n-1}\right)\right] \otimes\left(y_{n j} \cdot f_{n-1}\right) \in V_{n} \otimes V_{n+1}+V_{n+1} \otimes V_{n} .
\end{aligned}
$$

Therefore, $\Delta\left(V_{n}\right) \subset V_{n} \otimes V_{n+1}+V_{n+1} \otimes V_{n} \subset W \otimes W, n \geq 1$, and since $\Delta\left(V_{0}\right) \subset V_{1} \otimes V_{1} \subset W \otimes W$, one has $\Delta(W) \subset W \otimes W$, i.e. $W$ is a Lie subcoalgebra of $L^{\odot}$. 
Let $V$ be a Lie subcoalgebra of $L^{\odot}$. For any $h \in V$ and $x \in L$, one has $\Delta(h)=\sum_{j=1}^{n} h_{j}^{1} \otimes h_{j}^{2} \in V \otimes V$ and $x \cdot h=\gamma_{h}(x)=\sum_{j=1}^{n}\left\langle h_{j}^{1}, x\right\rangle h_{j}^{2} \in V$. Therefore, if $V$ contains $f$, one has $V_{0} \subset V$ and $V_{1}=\gamma_{f}(L) \subset V$. It is readily seen by induction that $V_{n} \subset V$ for all $n \geq 0$. It follows that $W=\sum_{n \geq 0} V_{n}$ is contained in $V$.

\section{Note.}

(i) One can prove, by induction, that the above $V_{n}, n \geq 0$, are finite dimensional.

(ii) One has for $n \geq 1, V_{n}=\operatorname{span}\left\{{ }^{t} a d x_{1} \circ{ }^{t} a d x_{2} \circ \ldots \circ{ }^{t} a d x_{n}(f)\right.$, $\left.x_{1}, \ldots, x_{n} \in L\right\}$.

Therefore, if $L$ is nilpotent of class $k$, then for any $f \in L^{\odot}$, the associated sequence of subspaces $\left(V_{n}\right)_{n \geq 0}$ is such that $V_{n}=(0)$, for $n \geq k$. It follows that $f$ belongs to the finite dimensional Lie subcoalgebra $W=\sum_{n=0}^{k-1} V_{n}$ of $L^{\odot}$. Hence, one has $L^{\odot}=\operatorname{Loc}\left(L^{\odot}\right)$ the sum of the finite dimensional Lie subcoalgebras of $L^{\odot}$. In particular, if $L$ is abelian, one has $V_{n}=(0)$, $n \geq 1$, and $L^{\odot}=L^{*}$.

More generally, one sees that $L^{\odot}=\operatorname{Loc}\left(L^{\odot}\right)$ iff for each $f \in L^{\odot}$ the above associated Lie subcoalgebra $W$ of $L^{\odot}$ is finite dimensional; in this case, there exists $k$ such that $W=\sum_{n=0}^{k} V_{n}$. Question : what is the class of all Lie algebras $L$ such that $L^{\odot}=\operatorname{Loc}\left(L^{\odot}\right)$ ?

\section{References}

1. W. Michaelis, Lie coalgebras, Advances in Math. 38 (1980), 1-54.

2. W. Michaelis, An example of a non-zero Lie coalgebra $M$ for which $\operatorname{Loc}(M)=(0)$, J. Pure Appl. Algebra 68 (1990), 341-348.

3. W. D. Nichols, The structure of the dual Lie coalgebra of the Witt algebra, J. Pure Appl. Algebra 68 (1990), 359-364.

4. W. D. Nichols, On Lie and associative duals, J. Pure Appl. Algebra 87 (1993), 313-320.

5. E. J. TAFT, Witt and Virasoro algebras as Lie bialgebras, J. Pure Appl. Algebra 87 (1993), 301-312. 
6. E. J. TAFT, Algebraic aspect of linearly recursive sequences, in "Advances in Hopf algebras," edited by J. Bergen, S. Montgomery, Marcel Dekker, New-York, 1994, pp. 299-317.

Keywords. Lie coalgebras

1991 Mathematics subject classifications: 16W30

\author{
Mathématiques Pures \\ Complexe Scientifique des Cézeaux \\ 63177 Aubière Cedex \\ FRANCE \\ e-mail: diarra@ucfma.univ-bpclermont.fr
}

Primera versió rebuda el 16 de Març de 1995, darrera versió rebuda el 17 de Maig de 1995 